\title{
Formation of Diethyl 2-Amino-1-cyclopentenylphosphonates - A Simple Synthesis with Unique Mechanism
}

Abed Al Aziz Al Quntar, ${ }^{a}$ Hemant Kumar Srivastava, ${ }^{a, b}$ Morris Srebnik, ${ }^{* a}$ Artem Melman, ${ }^{c}$ Rachel TaShma $^{a}$ and Avital Shurki* ${ }^{a, b}$

${ }^{a}$ Department of Medicinal Chemistry and Natural Products, School of Pharmacy, The Hebrew University of Jerusalem, Jerusalem, Israel. Fax: +972-2-6757076; +972-26758696; E-mail: msrebni@md.huji.ac.il,avital@md.huji.ac.il,

${ }^{b}$ The Lise-Meitner Minerva Center for Computational Quantum Chemistry, The Hebrew University of Jerusalem, Jerusalem, Israel

${ }^{c}$ Department of Organic Chemistry, Institute of Chemistry, Hebrew University of Jerusalem, Jerusalem, Israel

\section{Supporting Information}

\section{Table of Content}

Computational Part

Table $1 S$. Energy profiles of the proposed mechanism along with the two possible paths $\mathbf{a}$ and $\mathbf{b}$ for the first step.

Table $2 S$. Comparison of the energies obtained for $\mathrm{C}$ vs. $\mathrm{N}$ cyclization of enamine.

Table 3S. Energies (hartree) of all the structures presented in Figures-1\&2 S4

Table 4S. Cartesian coordinates of all the calculated structures $\quad$ S5

Experimental Part

Analytical data for 1a-k

${ }^{1} \mathrm{H}$ NMR and ${ }^{13} \mathrm{C}$ NMR spectra of 1a $\quad$ S14

$\begin{array}{lr}{ }^{1} \mathrm{H} \text { NMR and }{ }^{13} \mathrm{C} \text { NMR spectra of } \mathbf{1 b} & \mathrm{S} 15\end{array}$

${ }^{1} \mathrm{H}$ NMR and ${ }^{13} \mathrm{C}$ NMR spectra of 1c S16

$\begin{array}{ll}{ }^{1} \mathrm{H} \text { NMR and }{ }^{13} \mathrm{C} \text { NMR spectra of } \mathbf{1 d} & \text { S17 }\end{array}$

${ }^{1} \mathrm{H}$ NMR and ${ }^{13} \mathrm{C}$ NMR spectra of 1e S18

$\begin{array}{ll}{ }^{1} \mathrm{H} \text { NMR and }{ }^{13} \mathrm{C} \text { NMR spectra of } \mathbf{1 f} & \text { S19 }\end{array}$ 
${ }^{1} \mathrm{H}$ NMR and ${ }^{13} \mathrm{C}$ NMR spectra of $\mathbf{1 g} \quad$ S20

${ }^{1} \mathrm{H}$ NMR and ${ }^{13} \mathrm{C}$ NMR spectra of $\mathbf{1 h} \quad$ S21

${ }^{1} \mathrm{H}$ NMR and ${ }^{13} \mathrm{C}$ NMR spectra of $\mathbf{1 i} \quad$ S22

${ }^{1} \mathrm{H}$ NMR and ${ }^{13} \mathrm{C}$ NMR spectra of $\mathbf{1 j} \quad$ S23

${ }^{1} \mathrm{H}$ NMR and ${ }^{13} \mathrm{C}$ NMR spectra of $\mathbf{1 k} \quad$ S24 


\section{Computational Part}

Table 1S. Energy profiles of the proposed mechanism along with the two possible paths $\mathbf{a}$ and $\mathbf{b}$ for the first step. ${ }^{2}$

\begin{tabular}{|c|c|c|c|c|c|}
\hline Entry & Level $^{b}$ & TS- $1^{\mathrm{c}}$ & Int- ${ }^{c}$ & TS- $2^{\mathrm{c}}$ & Int- $2^{\mathrm{c}}$ \\
\hline \multicolumn{6}{|c|}{ Path a ( $Z$ intermediate) } \\
\hline 1 & B3LYP/6-31g* & 13.5 & 10.1 & 18.8 & -40.3 \\
\hline 2 & B3LYP/6-31+g** & 14.9 & 10.0 & 18.7 & -43.2 \\
\hline 3 & B3LYP/aug-cc-pvDZ & 13.2 & 7.4 & 16.1 & -44.8 \\
\hline \multicolumn{6}{|c|}{ Path b ( $E$ intermediate) } \\
\hline 1 & B3LYP/6-31g* & 18.8 & 18.3 & & \\
\hline 2 & B3LYP/6-31+g** & 19.6 & 18.5 & & \\
\hline 3 & B3LYP/aug-cc-pvDZ & 17.9 & 16.2 & & \\
\hline
\end{tabular}

Table 2S. Comparison of the energies obtained for C- vs. N-cyclization of enamine. $^{a}$

\begin{tabular}{|c|c|c|c|}
\hline Entry & Level $^{b}$ & TS- $3^{\mathrm{c}}$ & Int-3 \\
\hline \multicolumn{4}{|c|}{ C cyclization } \\
\hline 1 & B3LYP/6-31g* & 26.6 & -10.8 \\
\hline 2 & B3LYP/6-31+g** & 24.9 & -14.9 \\
\hline 3 & B3LYP/aug-cc-pvDZ & 24.9 & -14.6 \\
\hline \multicolumn{4}{|c|}{$\mathrm{N}$ cyclization } \\
\hline 1 & B3LYP/6-31g* & 23.1 & 2.1 \\
\hline 2 & B3LYP/6-31+g** & 22.3 & -1.9 \\
\hline 3 & B3LYP/aug-cc-pvDZ & 22.6 & -1.3 \\
\hline
\end{tabular}


an intermediate in the reaction, we would expect to obtain a mixture of both C- and Ncyclization with a ratio which favors the N-cyclization and not solely 2-Amino-1cyclopentenylphosphonate which is the product of C-cyclization.

Table 3S. Energies (hartree) of all the structures presented in Figures-1\&2

\begin{tabular}{ccccc}
\hline Structure & B3LYP/6-31G* & B3LYP/6-31+G** & B3LYP/AUG-cc-pVDZ & Img. \\
& OPT PCM & SP PCM & SP PCM & Freq. \\
\hline React (Figure-1a) & -1475.70231526 & -1475.76573833 & -1475.81920331 & $0^{\text {a }}$ \\
TS-a1 & -1475.68073356 & -1475.74203915 & -1475.79809843 & 1 \\
Int-a1 & -1475.68617255 & -1475.74894636 & -1475.80744987 & 0 \\
TS-a2 & -1475.67240930 & -1475.73506852 & -1475.79361012 & 1 \\
Int-a2 & -1475.76654149 & -1475.83384389 & -1475.89060959 & $0^{\text {a }}$ \\
React (Figure-1b) & -1475.70341396 & -1475.76684899 & -1475.82032178 & $0^{\text {a }}$ \\
TS-b1 & -1475.67341173 & -1475.73563706 & -1475.79186332 & 1 \\
Int-b1 & -1475.67427367 & -1475.73738657 & -1475.79454392 & $0^{\text {a }}$ \\
React (Figure-2a) & -1475.76338164 & -1475.82290156 & -1475.88012631 & 0 \\
TS3 & -1475.72092477 & -1475.78314940 & -1475.84048421 & 1 \\
Int-3 & -1475.78055294 & -1475.84665270 & -1475.90335675 & $0^{\text {a }}$ \\
React (Figure-2b) & -1475.76136090 & -1475.82003881 & -1475.87721332 & 0 \\
TS3 & -1475.72460506 & -1475.78452422 & -1475.84113379 & 1 \\
Int-3 & -1475.75802598 & -1475.82305297 & -1475.87935637 & $0^{\text {a }}$ \\
\hline
\end{tabular}

${ }^{a}$ very low negative frequency values which is negligible 


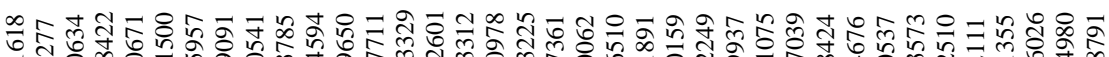

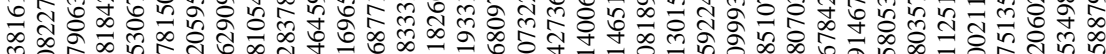

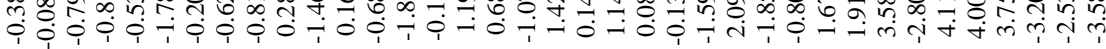

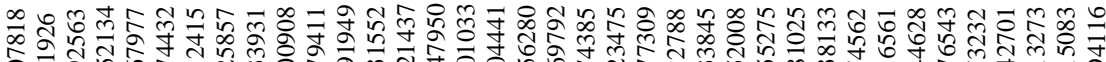

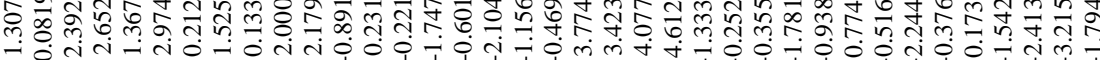

㓷

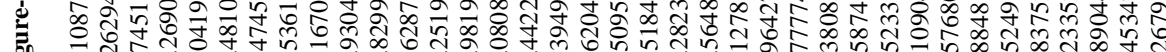

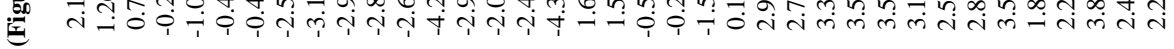

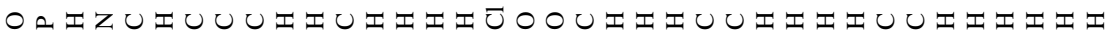

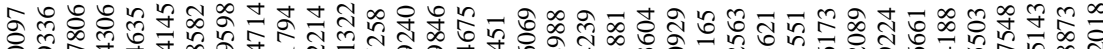

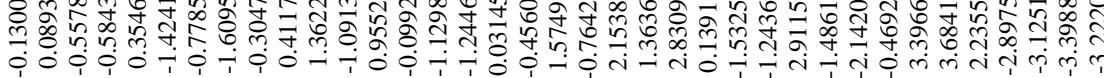

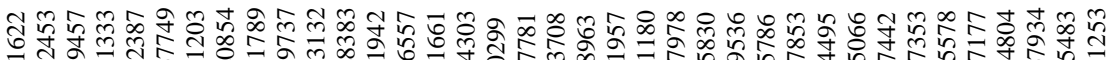

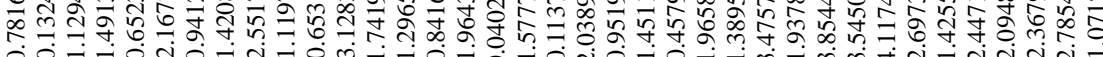

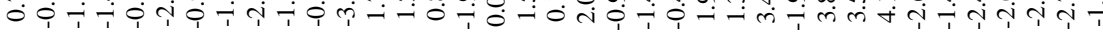

I

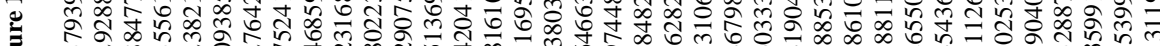

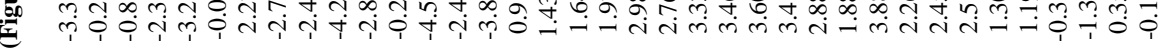

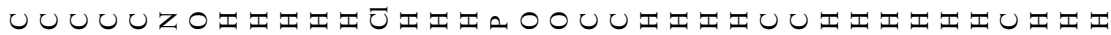

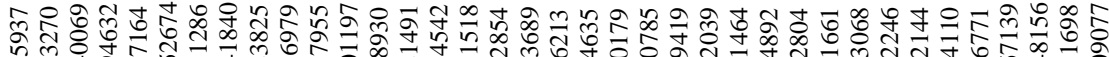

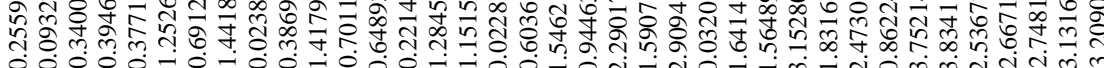

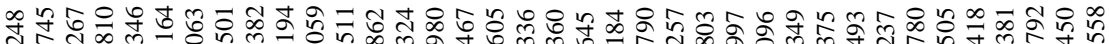

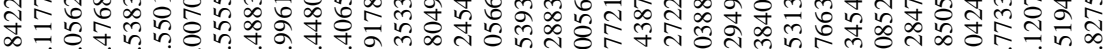

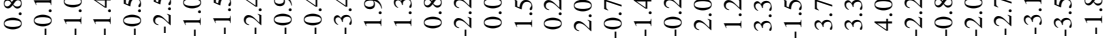

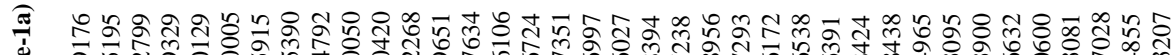
音

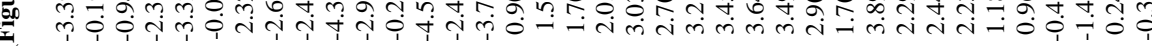

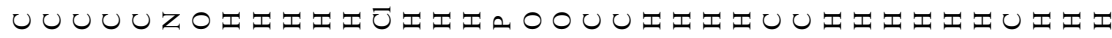

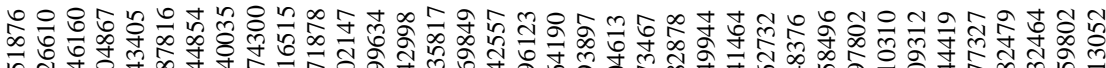

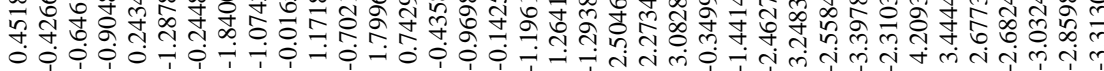

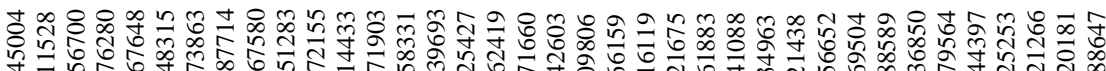

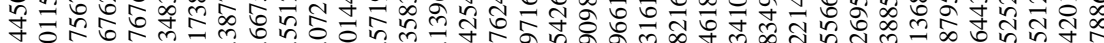

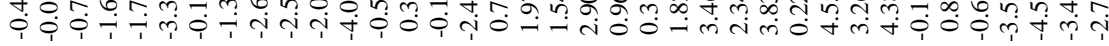

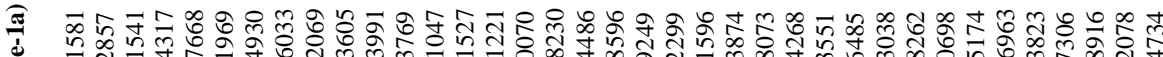

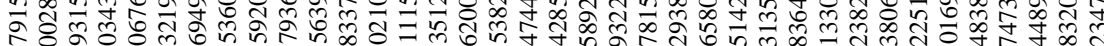

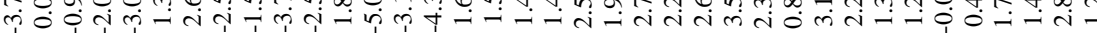




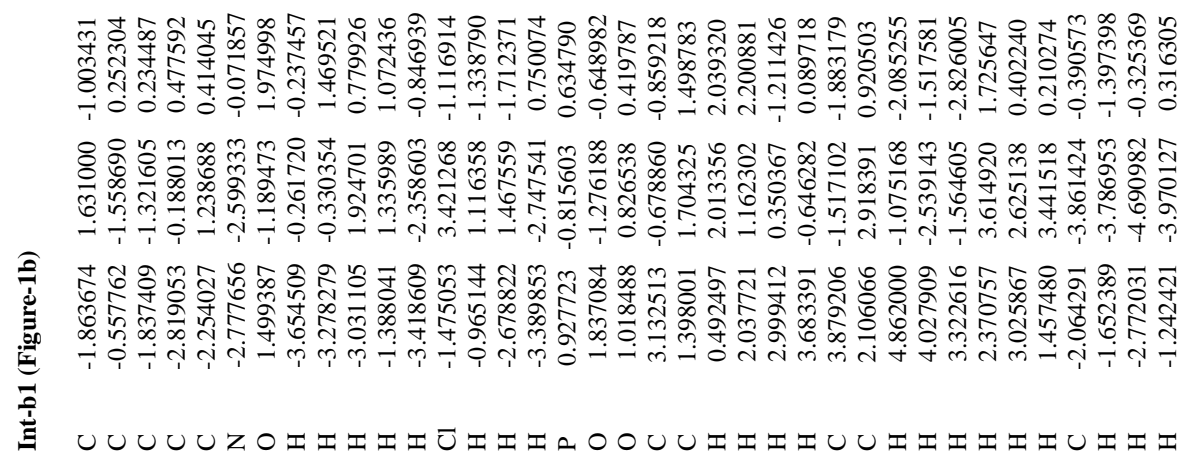

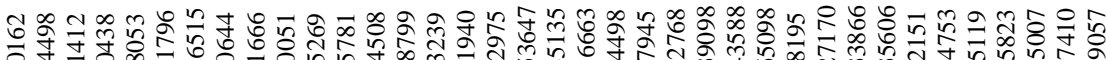

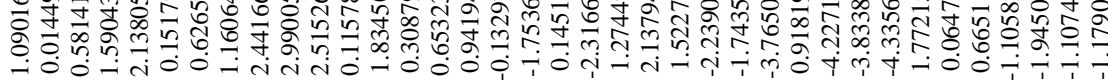

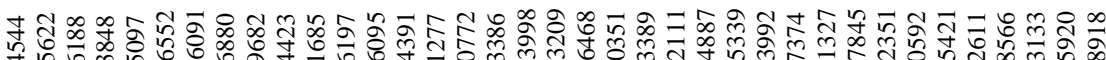

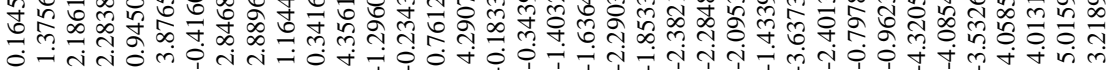

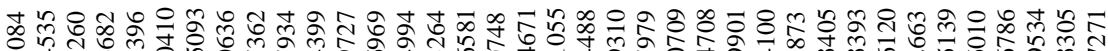

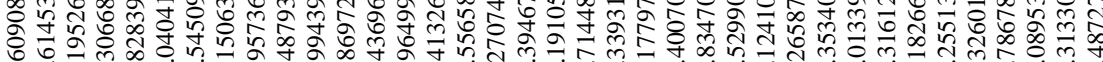

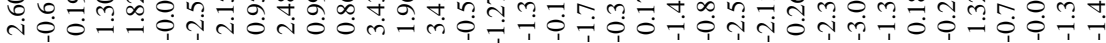

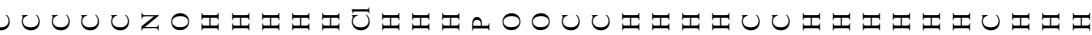

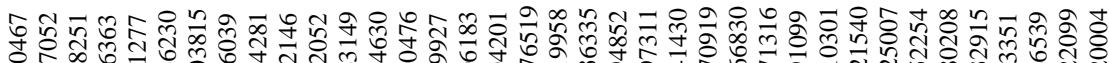

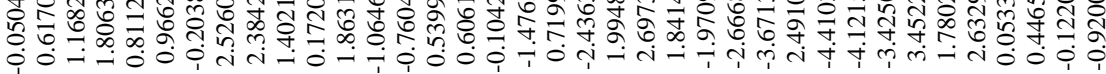

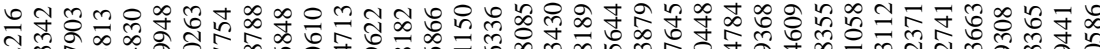

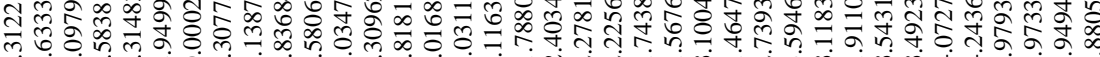

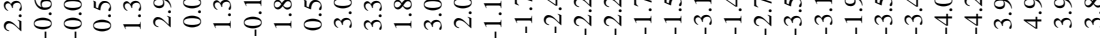

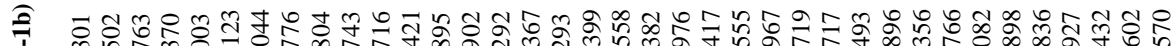
至

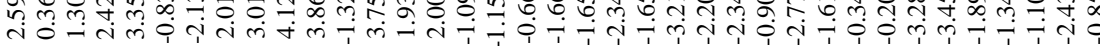

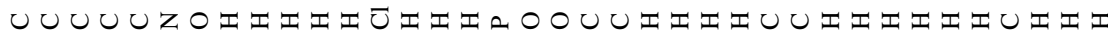

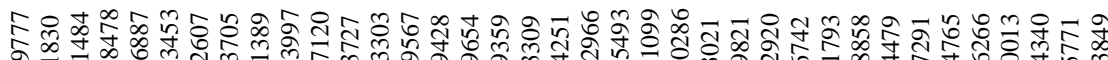

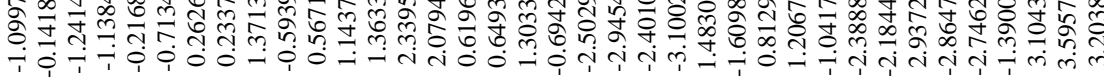

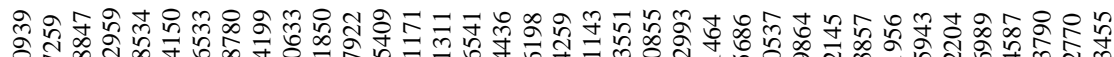

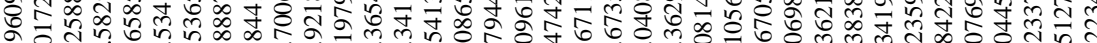

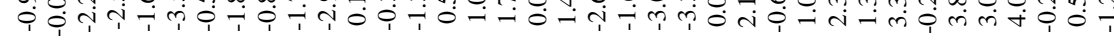

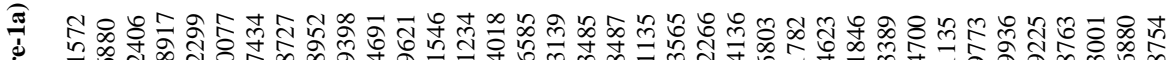

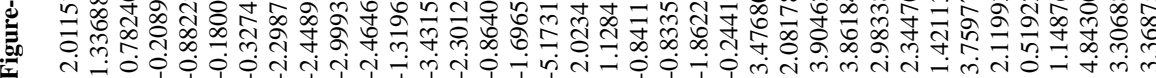




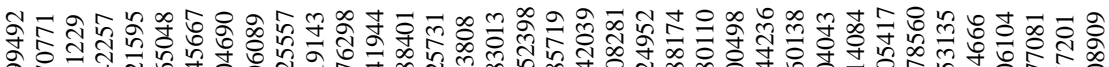

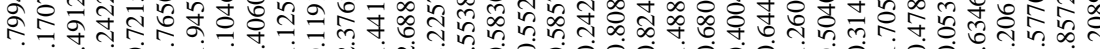

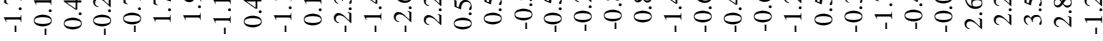

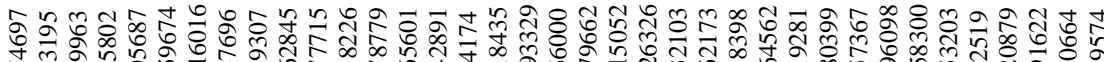

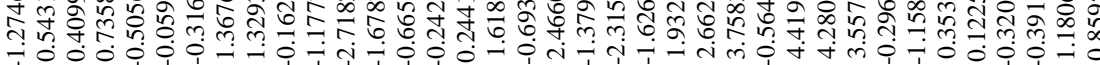

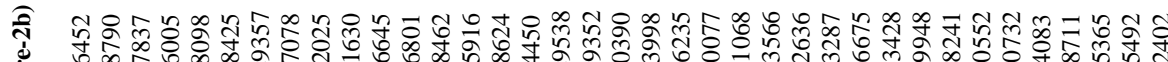

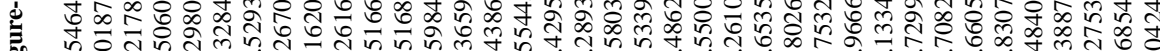

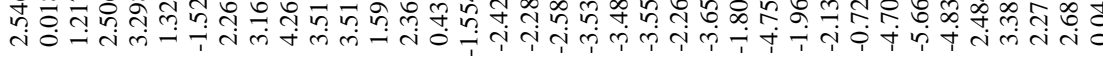

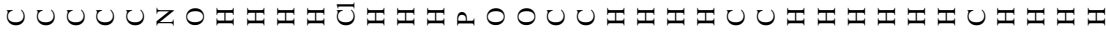

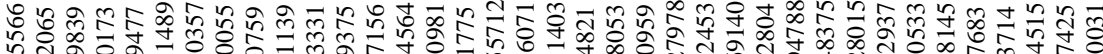

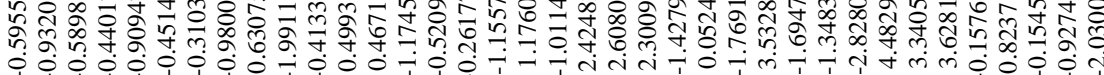

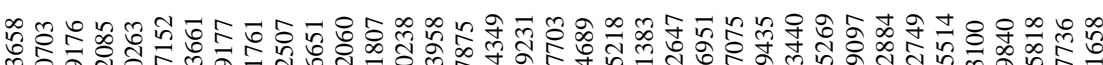
तु

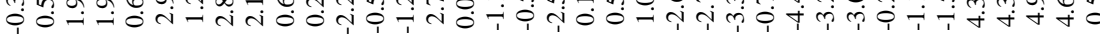

శᄑ

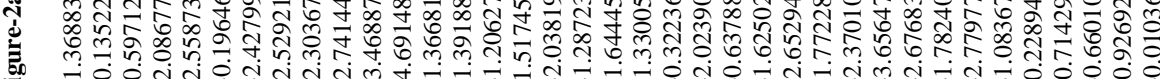

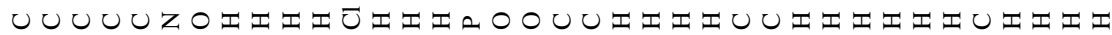

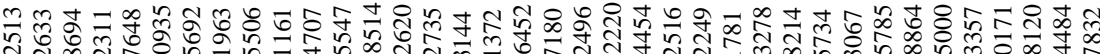

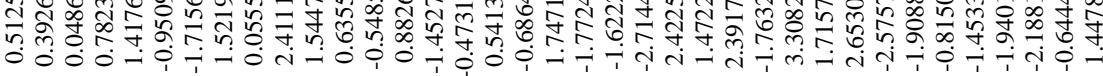

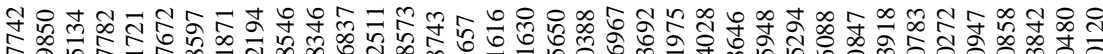

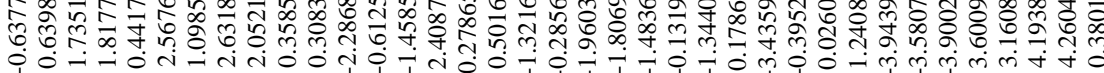

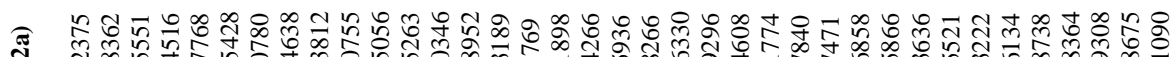

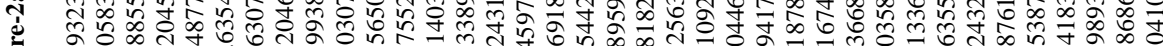

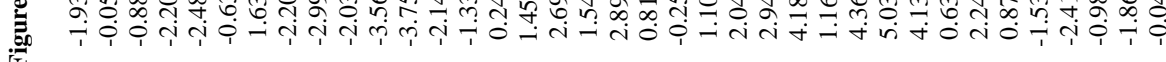

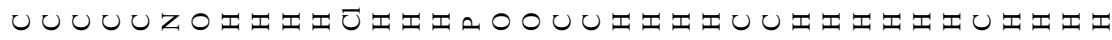

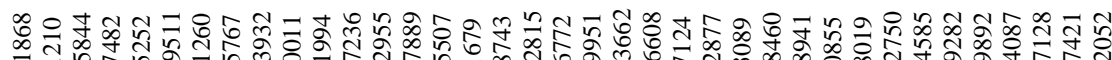

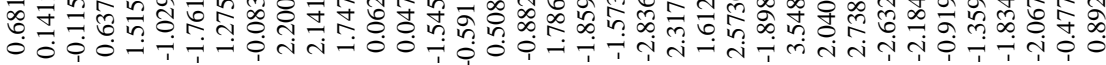

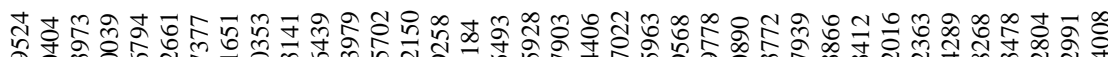

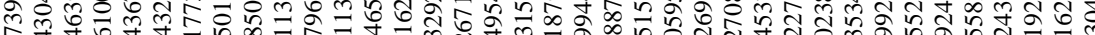

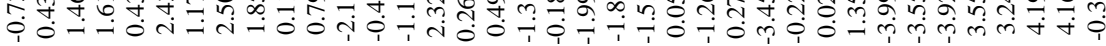

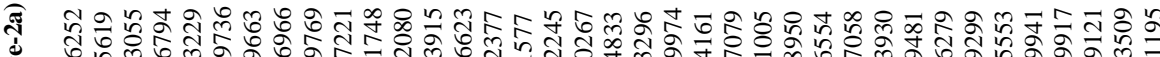

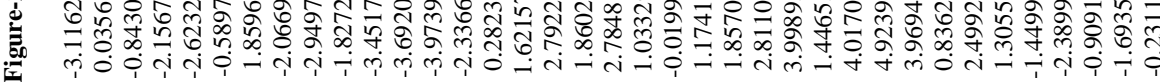
(1)

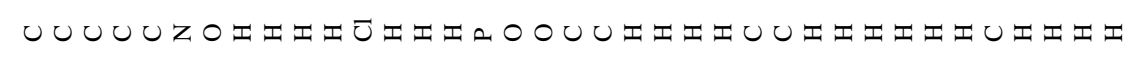




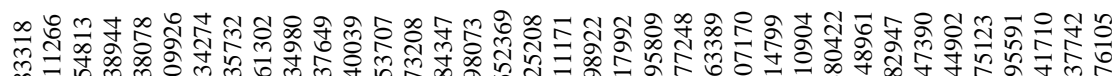

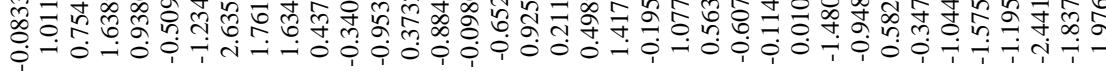

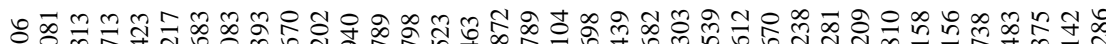

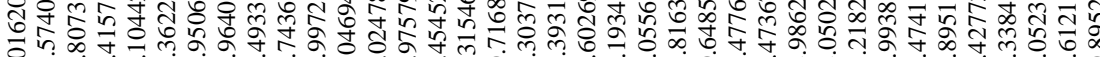

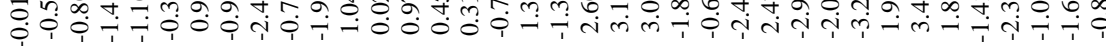

ลิ

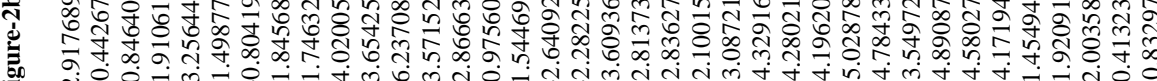

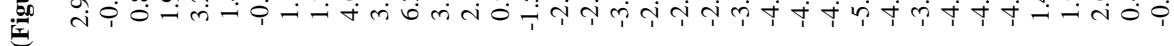

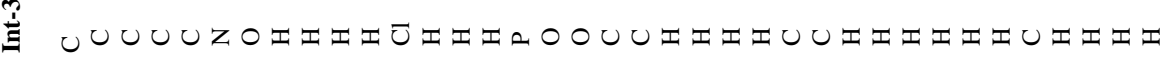

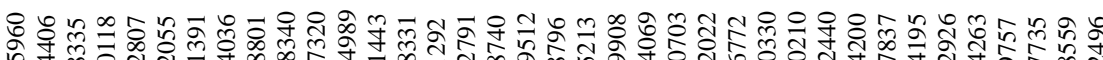

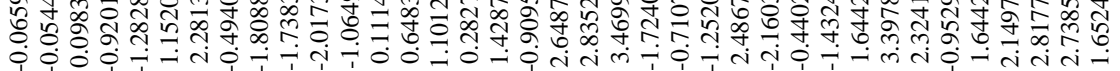

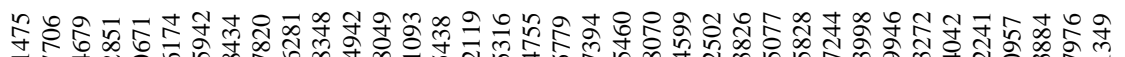

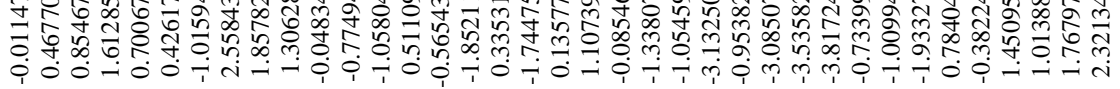

v)

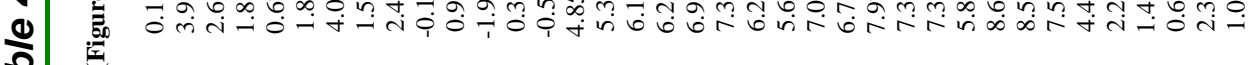




\section{Experimental Part}

1a: ${ }^{1} \mathrm{H}$ NMR $\left(300 \mathrm{MHz}\right.$, Acetone d): $\delta 1.88\left(\mathrm{t}, 6 \mathrm{H}, J_{\mathrm{HH}}=7.2 \mathrm{~Hz}\right), 1.93\left(\mathrm{qn}, 2 \mathrm{H}, J_{\mathrm{HH}}=\right.$ $7.2 \mathrm{~Hz}), 2.97\left(\mathrm{dt}, 2 \mathrm{H}, J_{\mathrm{HH}}=7.8 \mathrm{~Hz},{ }^{3} J_{\mathrm{PH}}=2.4 \mathrm{~Hz}\right), 3.41\left(\mathrm{t}, 2 \mathrm{H}, J_{\mathrm{HH}}=6.9 \mathrm{~Hz}\right), 3.87$ $\left(\mathrm{qn}, 4 \mathrm{H}, J_{\mathrm{HH}}=7.2 \mathrm{~Hz}\right), 4.42(\mathrm{~s}, 2 \mathrm{H}), 7.20-7.38$ (overlap, 5H). ${ }^{31} \mathrm{P}$ NMR (121.4 MHZ, Acetone d): $\delta 27.85 ;{ }^{13} \mathrm{C}$ NMR $\left(75.5 \mathrm{MHz}\right.$, Acetone d): $16.1\left(\mathrm{~d},{ }^{3} J_{\mathrm{PC}}=6.9 \mathrm{~Hz}\right), 21.3$, $32.1,49.5,52.6,60.0\left(\mathrm{~d},{ }^{2} J_{\mathrm{PC}}=5.2 \mathrm{~Hz}\right), 127.3,127.4,128.8,137.2,164.5\left(\mathrm{~d},{ }^{2} J_{\mathrm{PC}}=\right.$ $20.1 \mathrm{~Hz}) ; \mathrm{MS}(\mathrm{EI}): \mathrm{m} / \mathrm{z}$ (\%) 309 (8.5), 264 (2.1), 207 (1.1), 172 (100), 144 (6.0), 104 (10.3), 91 (80.1), 77 (1.2), 65 (21.3), 41 (10.3); Anal. Calcd for $\mathrm{C}_{16} \mathrm{H}_{24} \mathrm{NO}_{3} \mathrm{P}: \mathrm{C}$, 62.12; H, 7.82; N, 4.53; P, 10.01. Found: C, 62.01; H, 7.89; N, 4.49; P, 10.09.

1b: ${ }^{1} \mathrm{H}$ NMR (300 MHz, Methanol d): $\delta 1.18,\left(\mathrm{~d}, 6 \mathrm{H}, J_{\mathrm{HH}}=6.6 \mathrm{~Hz}\right), 1.31\left(\mathrm{t}, 6 \mathrm{H}, J_{\mathrm{HH}}\right.$ $=6.6 \mathrm{~Hz}), 1.89\left(\mathrm{qn}, 2 \mathrm{H}, J_{\mathrm{HH}}=7.2 \mathrm{~Hz}\right), 2.82\left(\mathrm{dt}, 2 \mathrm{H}, J_{\mathrm{HH}}=8.1 \mathrm{~Hz},{ }^{3} J_{\mathrm{PH}}=1.8 \mathrm{~Hz}\right)$, $3.36\left(\mathrm{t}, 2 \mathrm{H}, J_{\mathrm{HH}}=6.9 \mathrm{~Hz}\right), 3.84(\mathrm{~m}, 1 \mathrm{H}), 3.97\left(\mathrm{qn}, 4 \mathrm{H}, J_{\mathrm{HH}}=7.2 \mathrm{~Hz}\right) \cdot{ }^{31} \mathrm{P} \mathrm{NMR}(121.4$ MHZ, Methanol d): $\delta 31.67 ;{ }^{13} \mathrm{C}$ NMR $\left(75.5 \mathrm{MHz}\right.$, Methanol d): $15.7\left(\mathrm{~d},{ }^{3} J_{\mathrm{PC}}=7.2\right.$ $\mathrm{Hz}), 18.0,19.8,20.8,32.5,45.6,60.9\left(\mathrm{~d},{ }^{2} J_{\mathrm{PC}}=5.4 \mathrm{~Hz}\right), 164.7\left(\mathrm{~d},{ }^{2} J_{\mathrm{PC}}=20.9 \mathrm{~Hz}\right)$; MS(EI):m/z (\%) 261 (12.7), 246 (16.7), 216 (4.9), 146 (8.8), 124 (100), 96 (28.4), 83 (37.3), 41 (14.7); Anal. Calcd for $\mathrm{C}_{12} \mathrm{H}_{24} \mathrm{NO}_{3} \mathrm{P}: \mathrm{C}, 55.16 ; \mathrm{H}, 9.26 ; \mathrm{N}, 5.36 ; \mathrm{P}, 11.85$. Found: C, 55.20; H, 9.19; N, 5.43; P, 11.81.

1c: ${ }^{1} \mathrm{H}$ NMR (300 MHz, Methanol d): $\delta 0.93\left(\mathrm{t}, 3 \mathrm{H}, J_{\mathrm{HH}}=7.5 \mathrm{~Hz}\right), 1.28\left(\mathrm{t}, 6 \mathrm{H}, J_{\mathrm{HH}}=\right.$ $7.2 \mathrm{~Hz}), 1.68(\mathrm{~m}, 2 \mathrm{H}), 1.92\left(\mathrm{qn}, 2 \mathrm{H}, J_{\mathrm{HH}}=6.9 \mathrm{~Hz}\right), 2.83\left(\mathrm{dt}, 2 \mathrm{H}, J_{\mathrm{HH}}=7.5 \mathrm{~Hz},{ }^{3} J_{\mathrm{PH}}=\right.$ $2.1 \mathrm{~Hz}), 3.16\left(\mathrm{t}, 2 \mathrm{H}, J_{\mathrm{HH}}=7.2 \mathrm{~Hz}\right), 3.42\left(\mathrm{t}, 2 \mathrm{H}, J_{\mathrm{HH}}=6.6 \mathrm{~Hz}\right), 3.95\left(\mathrm{qn}, 4 \mathrm{H}, J_{\mathrm{HH}}=7.2\right.$ Hz). ${ }^{31} \mathrm{P}$ NMR (121.4 MHZ, Methanol d): $\delta 31.78 ;{ }^{13} \mathrm{C}$ NMR $(75.5 \mathrm{MHz}$, Methanol d): 10. 8, $15.7\left(\mathrm{~d},{ }^{3} J_{\mathrm{PC}}=6.8 \mathrm{~Hz}\right), 20.8,21.0,32.3,41.2,52.4,60.9\left(\mathrm{~d},{ }^{2} J_{\mathrm{PC}}=5.4 \mathrm{~Hz}\right)$, $165.4\left(\mathrm{~d},{ }^{2} J_{\mathrm{PC}}=20.9 \mathrm{~Hz}\right) ; \operatorname{MS}(\mathrm{EI}): \mathrm{m} / \mathrm{z}(\%) 261$ (16.7), 232 (26.5), 216 (8.8), 204 
(8.8), 146 (17.6), 124 (100), 110 (42.2), 97 (68.6), 83 (68.6), 55 (29.9), 41 (55.9);

Anal. Calcd for $\mathrm{C}_{12} \mathrm{H}_{24} \mathrm{NO}_{3} \mathrm{P}: \mathrm{C}, 55.16 ; \mathrm{H}, 9.26 ; \mathrm{N}, 5.36 ; \mathrm{P}, 11.85$. Found: C, 55.03; H, 9.20; N, 5.46; P, 11.94 .

1d: ${ }^{1} \mathrm{H}$ NMR $\left(300 \mathrm{MHz}\right.$, Methanol d): $\delta 0.91\left(\mathrm{t}, 3 \mathrm{H}, J_{\mathrm{HH}}=6.6 \mathrm{~Hz}\right), 1.35\left(\mathrm{t}, 6 \mathrm{H}, J_{\mathrm{HH}}\right.$ $=7.2 \mathrm{~Hz}), 1.20-1.70($ overlap, $6 \mathrm{H}), 1.93\left(\mathrm{qn}, 2 \mathrm{H}, J_{\mathrm{HH}}=7.5 \mathrm{~Hz}\right), 2.83\left(\mathrm{dt}, 2 \mathrm{H}, J_{\mathrm{HH}}=\right.$ $\left.7.8 \mathrm{~Hz},{ }^{3} J_{\mathrm{PH}}=2.1 \mathrm{~Hz}\right), 3.19\left(\mathrm{t}, 2 \mathrm{H}, J_{\mathrm{HH}}=7.8 \mathrm{~Hz}\right), 3.42\left(\mathrm{t}, 2 \mathrm{H}, J_{\mathrm{HH}}=7.2 \mathrm{~Hz}\right), 3.93(\mathrm{qn}$, $\left.4 \mathrm{H}, J_{\mathrm{HH}}=7.2 \mathrm{~Hz}\right) .{ }^{31} \mathrm{P}$ NMR $\left(121.4 \mathrm{MHZ}\right.$, Methanol d): $\delta 30.03 ;{ }^{13} \mathrm{C}$ NMR $(75.5$ MHz, Methanol d): 13.4, $15.7\left(\mathrm{~d},{ }^{3} J_{\mathrm{PC}}=7.2 \mathrm{~Hz}\right), 21.0,22.3,25.8,28.8,40.3,45.8$, $52.4,60.9\left(\mathrm{~d},{ }^{2} J_{\mathrm{PC}}=5.1 \mathrm{~Hz}\right), 165.4\left(\mathrm{~d},{ }^{2} J_{\mathrm{PC}}=20.9 \mathrm{~Hz}\right) ; \mathrm{MS}(\mathrm{EI}): \mathrm{m} / \mathrm{z}(\%) 289(2.2)$, 260 (17.0), 246 (9.1), 233 (36.4), 152 (51.1), 138 (92.2), 79 (100), 83 (11.4), 55 (22.7), 41 (52.3); Anal. Calcd for $\mathrm{C}_{14} \mathrm{H}_{28} \mathrm{NO}_{3} \mathrm{P}: \mathrm{C}, 58.11 ; \mathrm{H}, 9.75 ; \mathrm{N}, 4.84 ; \mathrm{P}, 10.70$. Found: C, 58.23; H, 9.80; N, 4.76; P, 10.61.

1e: ${ }^{1} \mathrm{H}$ NMR (300 MHz, Methanol d): $\delta 1.28\left(\mathrm{t}, 6 \mathrm{H}, J_{\mathrm{HH}}=6.9 \mathrm{~Hz}\right), 1.40-1.70$ (overlap, $10 \mathrm{H}), 1.88\left(\mathrm{qn}, 2 \mathrm{H}, J_{\mathrm{HH}}=7.2 \mathrm{~Hz}\right), 2.81\left(\mathrm{dt}, 2 \mathrm{H}, J_{\mathrm{HH}}=7.5 \mathrm{~Hz},{ }^{3} J_{\mathrm{PH}}=2.1 \mathrm{~Hz}\right), 3.06$, $(\mathrm{m}, 1 \mathrm{H}), 3.37\left(\mathrm{t}, 2 \mathrm{H}, J_{\mathrm{HH}}=6.9 \mathrm{~Hz}\right), 3.95\left(\mathrm{qn}, 4 \mathrm{H}, J_{\mathrm{HH}}=7.5 \mathrm{~Hz}\right) .{ }^{31} \mathrm{P}$ NMR $(121.4$ MHZ, Methanol d): $\delta 30.08 ;{ }^{13} \mathrm{C}$ NMR $\left(75.5 \mathrm{MHz}\right.$, Methanol d): $15.7\left(\mathrm{~d},{ }^{3} J_{\mathrm{PC}}=7.2\right.$ $\mathrm{Hz}), 20.9,24.3,30.7,32.4,41.2,46.8,54.2,60.9\left(\mathrm{~d},{ }^{2} J_{\mathrm{PC}}=5.1 \mathrm{~Hz}\right), 165.5\left(\mathrm{~d},{ }^{2} J_{\mathrm{PC}}=\right.$ 21.2 Hz); MS(EI):m/z (\%) 301 (8.8), 256 (5.9), 220 (100), 192 (26.5), 164 (82.3), 146 (41.2), 83 (55.8), 55 (41.2), 41 (52.9); Anal. Calcd for $\mathrm{C}_{15} \mathrm{H}_{28} \mathrm{NO}_{3} \mathrm{P}: \mathrm{C}, 59.78 ; \mathrm{H}$, 9.36; N, 4.65; P, 10.28. Found: C, 59.89; H, 9.29; N, 4.71; P, 10.18 .

1f: ${ }^{1} \mathrm{H}$ NMR (300 MHz, Methanol d): $\delta 1.23\left(\mathrm{t}, 6 \mathrm{H}, J_{\mathrm{HH}}=7.2 \mathrm{~Hz}\right), 1.85\left(\mathrm{qn}, 2 \mathrm{H}, J_{\mathrm{HH}}=\right.$ $7.5 \mathrm{~Hz}), 2.70(\mathrm{~s}, 3 \mathrm{H}), 2.84\left(\mathrm{dt}, 2 \mathrm{H}, J_{\mathrm{HH}}=7.8 \mathrm{~Hz},{ }^{3} J_{\mathrm{PH}}=1.8 \mathrm{~Hz}\right), 3.28\left(\mathrm{t}, 2 \mathrm{H}, J_{\mathrm{HH}}=7.2\right.$ $\mathrm{Hz}), 3.93\left(\mathrm{qn}, 4 \mathrm{H}, J_{\mathrm{HH}}=6.9 \mathrm{~Hz}\right) \cdot{ }^{31} \mathrm{P}$ NMR (121.4 MHZ, Methanol d): $\delta 31.17 ;{ }^{13} \mathrm{C}$ 
NMR (75.5 MHz, Methanol d): $15.7\left(\mathrm{~d},{ }^{3} J_{\mathrm{PC}}=6.9 \mathrm{~Hz}\right), 20.9,24.4,32.1,54.2,60.9(\mathrm{~d}$, $\left.{ }^{2} J_{\mathrm{PC}}=5.1 \mathrm{~Hz}\right), 166.0\left(\mathrm{~d},{ }^{2} J_{\mathrm{PC}}=20.2 \mathrm{~Hz}\right) ; \mathrm{MS}(\mathrm{EI}): \mathrm{m} / \mathrm{z}(\%) 233$ (13.7), 207 (1.1), 188 (2.2), 160 (6.8), 97 (100), 69 (12.7), 55 (10.8), 42 (23.5); Anal. Calcd for $\mathrm{C}_{10} \mathrm{H}_{20} \mathrm{NO}_{3} \mathrm{P}: \mathrm{C}, 51.49 ; \mathrm{H}, 8.64 ; \mathrm{N}, 6.01 ; 13.28$. Found: C, 51.61; H, 8.58; N, 5.90; P, 13.33.

1g: ${ }^{1} \mathrm{H}$ NMR (300 MHz, Methanol d): $\delta 1.14\left(\mathrm{~d}, 3 \mathrm{H}, J_{\mathrm{HH}}=6.9 \mathrm{~Hz}\right), 1.28\left(\mathrm{t}, 6 \mathrm{H}, J_{\mathrm{HH}}=\right.$ $7.2 \mathrm{~Hz}), 1.89\left(\mathrm{qn}, 1 \mathrm{H}, J_{\mathrm{HH}}=7.5 \mathrm{~Hz}\right), 1.90\left(\mathrm{qn}, 1 \mathrm{H}, J_{\mathrm{HH}}=7.5 \mathrm{~Hz}\right), 2.82\left(\mathrm{dt}, 1 \mathrm{H}, J_{\mathrm{HH}}=\right.$ $\left.7.8 \mathrm{~Hz},{ }^{3} J_{\mathrm{PH}}=1.8 \mathrm{~Hz}\right), 2.85\left(\mathrm{dt}, 1 \mathrm{H}, J_{\mathrm{HH}}=7.8 \mathrm{~Hz},{ }^{3} J_{\mathrm{PH}}=1.8 \mathrm{~Hz}\right), 3.39\left(\mathrm{t}, 1 \mathrm{H}, J_{\mathrm{HH}}=\right.$ $7.8 \mathrm{~Hz}), 3.43\left(\mathrm{t}, 1 \mathrm{H}, J_{\mathrm{HH}}=7.8 \mathrm{~Hz}\right), 3.58\left(\mathrm{~d}, 1 \mathrm{H}, J_{\mathrm{HH}}=6.3 \mathrm{~Hz}\right), 3.59\left(\mathrm{~d}, 1 \mathrm{H}, J_{\mathrm{HH}}=6.3\right.$ $\mathrm{Hz}), 3.73\left(\mathrm{qn}, 1 \mathrm{H}, J_{\mathrm{HH}}=6.3 \mathrm{~Hz}\right), 3.96\left(\mathrm{qn}, 4 \mathrm{H}, J_{\mathrm{HH}}=7.2 \mathrm{~Hz}\right) .{ }^{31} \mathrm{P} \mathrm{NMR}(121.4 \mathrm{MHZ}$, Methanol d): $\delta 32.28 ;{ }^{13} \mathrm{C}$ NMR $\left(75.5 \mathrm{MHz}\right.$, Methanol d): $12.4,15.5\left(\mathrm{~d},{ }^{3} J_{\mathrm{PC}}=6.9\right.$ $\mathrm{Hz}), 20.7,32.4,47.0,51.8,60.9\left(\mathrm{~d},{ }^{2} J_{\mathrm{PC}}=5.1 \mathrm{~Hz}\right), 62.7,165.7\left(\mathrm{~d},{ }^{2} J_{\mathrm{PC}}=20.6 \mathrm{~Hz}\right)$; MS(EI):m/z (\%) 277 (7.8), 246 (20.6), 220 (88.2), 192 (24.5), 164 (37.3), 146 (50.0), 126 (100), 122 (81.4), 110 (82.4), 80 (44.1), 41 (73.5); Anal. Calcd for $\mathrm{C}_{12} \mathrm{H}_{24} \mathrm{NO}_{4} \mathrm{P}$ : C, 51.98; H, 8.72; N, 5.05; P, 11.17. Found: C, 52.07; H, 8.81; N, 4.94; P, 11.10.

1h: ${ }^{1} \mathrm{H}$ NMR (300 MHz, Methanol d): $\delta 1.28\left(\mathrm{~d}, 6 \mathrm{H}, J_{\mathrm{HH}}=7.2 \mathrm{~Hz}\right), 1.90\left(\mathrm{qn}, 1 \mathrm{H}, J_{\mathrm{HH}}\right.$ $=7.9 \mathrm{~Hz}), 2.90\left(\mathrm{dt}, 2 \mathrm{H}, J_{\mathrm{HH}}=7.5 \mathrm{~Hz},{ }^{3} J_{\mathrm{PH}}=1.8 \mathrm{~Hz}\right), 3.31\left(\mathrm{t}, 2 \mathrm{H}, J_{\mathrm{HH}}=7.2 \mathrm{~Hz}\right), 3.45$ $\left(\mathrm{t}, 2 \mathrm{H}, J_{\mathrm{HH}}=7.2 \mathrm{~Hz}\right), 3.76\left(\mathrm{t}, 2 \mathrm{H}, J_{\mathrm{HH}}=5.7 \mathrm{~Hz}\right), 3.98\left(\mathrm{qn}, 4 \mathrm{H}, J_{\mathrm{HH}}=7.2 \mathrm{~Hz}\right) \cdot{ }^{31} \mathrm{P}$ NMR (121.4 MHZ, $\left.\mathrm{CDCl}_{3}\right): \delta 29.66 ;{ }^{13} \mathrm{C}$ NMR (75.5 MHz, Methanol d): $15.7\left(\mathrm{~d},{ }^{3} J_{\mathrm{PC}}\right.$ $=6.9 \mathrm{~Hz}), 21.1,32.3,43.0,58.1,61.1\left(\mathrm{~d},{ }^{2} J_{\mathrm{PC}}=5.5 \mathrm{~Hz}\right), 62.0,165.7\left(\mathrm{~d},{ }^{2} J_{\mathrm{PC}}=20.6\right.$ Hz); MS(EI):m/z (\%) 263 (13.7), 246 (2.9), 232 (15.7), 220 (98.0), 192 (25.5), 164 (40.2), 146 (46.1), 108 (100), 97 (45.1), 83 (27.5), 65 (14.7), 41 (17.6); 
Anal. Calcd for $\mathrm{C}_{11} \mathrm{H}_{22} \mathrm{NO}_{4} \mathrm{P}: \mathrm{C}, 50.18 ; \mathrm{H}, 8.42 ; \mathrm{N}, 5.32 ; \mathrm{P}, 11.76$. Found: C, 50.07; $\mathrm{H}, 8.38 ; \mathrm{N}, 5.38 ; \mathrm{P}, 11.85$.

1i: ${ }^{1} \mathrm{H}$ NMR (300 MHz, Methanol d): $\delta 1.29\left(\mathrm{~d}, 6 \mathrm{H}, J_{\mathrm{HH}}=6.9 \mathrm{~Hz}\right), 1.91\left(\mathrm{qn}, 1 \mathrm{H}, J_{\mathrm{HH}}\right.$ $=7.5 \mathrm{~Hz}), 2.84\left(\mathrm{dt}, 2 \mathrm{H}, J_{\mathrm{HH}}=7.8 \mathrm{~Hz},{ }^{3} J_{\mathrm{PH}}=2.1 \mathrm{~Hz}\right), 3.31(\mathrm{~s}, 3 \mathrm{H}), 3.33\left(\mathrm{t}, 2 \mathrm{H}, J_{\mathrm{HH}}=\right.$ $5.1 \mathrm{~Hz}), 3.49\left(\mathrm{t}, 2 \mathrm{H}, J_{\mathrm{HH}}=7.2 \mathrm{~Hz}\right), 3.56\left(\mathrm{t}, 2 \mathrm{H}, J_{\mathrm{HH}}=5.4 \mathrm{~Hz}\right), 3.96\left(\mathrm{qn}, 4 \mathrm{H}, J_{\mathrm{HH}}=7.2\right.$ Hz). ${ }^{31} \mathrm{P}$ NMR (121.4 MHZ, Methanol d): $\delta 31.37 ;{ }^{13} \mathrm{C}$ NMR $(75.5 \mathrm{MHz}$, Methanol d): $15.7\left(\mathrm{~d},{ }^{3} J_{\mathrm{PC}}=6.9 \mathrm{~Hz}\right), 21.1,32.3,45.7,53.2,58.1,61.0\left(\mathrm{~d},{ }^{2} J_{\mathrm{PC}}=5.4 \mathrm{~Hz}\right), 69.1$, $165.4\left(\mathrm{~d},{ }^{2} J_{\mathrm{PC}}=20.9 \mathrm{~Hz}\right) ; \operatorname{MS}(\mathrm{EI}): \mathrm{m} / \mathrm{z}(\%) 277$ (6.9), 262 (16.7), 220 (17.6), 146 (22.5), 108 (100), 96 (29.4), 83 (47.1), 58 (40.0), 41 (31.4); Anal. Calcd for $\mathrm{C}_{12} \mathrm{H}_{24} \mathrm{NO}_{4} \mathrm{P}: \mathrm{C}, 51.98 ; \mathrm{H}, 8.72 ; \mathrm{N}, 5.05 ; \mathrm{P}, 11.17$. Found: C, 52.08; H, 8.67; N, 4.94; $\mathrm{P}, 11.23$.

1j: ${ }^{1} \mathrm{H}$ NMR $\left(300 \mathrm{MHz}, \mathrm{CDCl}_{3}\right): \delta 1.30\left(\mathrm{t}, 6 \mathrm{H}, J_{\mathrm{HH}}=6.0 \mathrm{~Hz}\right), 1.74-1.87$ (overlap, $5 \mathrm{H}), 2.40-2.60($ overlap, $4 \mathrm{H}), 2.64\left(\right.$ broad t $\left., 2 \mathrm{H}, J_{\mathrm{HH}}=7.8 \mathrm{~Hz}\right), 3.25\left(\right.$ broad t, $2 \mathrm{H}, J_{\mathrm{HH}}$ $=5.4 \mathrm{~Hz}), 4.02\left(\mathrm{qn}, 4 \mathrm{H}, J_{\mathrm{HH}}=6.9 \mathrm{~Hz}\right) .{ }^{31} \mathrm{P} \mathrm{NMR}\left(121.4 \mathrm{MHZ}, \mathrm{CDCl}_{3}\right): \delta 28.23 ;{ }^{13} \mathrm{C}$ $\operatorname{NMR}\left(75.5 \mathrm{MHz}, \mathrm{CDCl}_{3}\right): 16.0\left(\mathrm{~d},{ }^{3} J_{\mathrm{PC}}=6.9 \mathrm{~Hz}\right), 22.9,27.8,29.4\left(\mathrm{~d},{ }^{2} J_{\mathrm{PC}}=4.3 \mathrm{~Hz}\right)$, $53.4,55.7,60.1\left(\mathrm{~d},{ }^{2} J_{\mathrm{PC}}=5.4 \mathrm{~Hz}\right), 71.8\left(\mathrm{~d},{ }^{1} J_{\mathrm{PC}}=217.5 \mathrm{~Hz}\right), 162.3\left(\mathrm{~d},{ }^{2} J_{\mathrm{PC}}=20.9\right.$ Hz); MS(EI):m/z (\%) 273 (3.7), 260 (5.3), 247 (7.8), 152 (18.4), 110 (100), 84 (19.8), 70 (28.3), 55 (8.6), 41 (9.5); Anal. Calcd for $\mathrm{C}_{13} \mathrm{H}_{24} \mathrm{NO}_{3} \mathrm{P}: \mathrm{C}, 57.13 ; \mathrm{H}, 8.85 ; \mathrm{N}, 5.12$; P, 11.33. Found: C, 57.21; H, 8.88; N, 5.04; P, 11.40.

1k: ${ }^{1} \mathrm{H}$ NMR $\left(300 \mathrm{MHz}, \mathrm{CDCl}_{3}\right): \delta 1.30\left(\mathrm{t}, 6 \mathrm{H}, J_{\mathrm{HH}}=7.2 \mathrm{~Hz}\right.$ ), 1.50-1.80 (overlap, 8H), 2.24-2.42 (overlap, 4H), $2.63\left(\right.$ broad t, $\left.2 \mathrm{H}, J_{\mathrm{HH}}=7.2 \mathrm{~Hz}\right), 3.25($ broad dt, $2 \mathrm{H}$, $\left.J_{\mathrm{HH}}=5.7 \mathrm{~Hz},{ }^{3} J_{\mathrm{PH}}=1.5 \mathrm{~Hz}\right), 4.02\left(\mathrm{qn}, 4 \mathrm{H}, J_{\mathrm{HH}}=6.9 \mathrm{~Hz}\right) .{ }^{31} \mathrm{P} \mathrm{NMR}(121.4 \mathrm{MHZ}$, 
$\left.\mathrm{CDCl}_{3}\right): \delta 28.62 ;{ }^{13} \mathrm{C} \mathrm{NMR}\left(75.5 \mathrm{MHz}, \mathrm{CDCl}_{3}\right): 15.6\left(\mathrm{~d},{ }^{3} \mathrm{~J}_{\mathrm{PC}}=6.9 \mathrm{~Hz}\right), 23.6,24.7$, $25.1,27.8\left(\mathrm{~d},{ }^{2} J_{\mathrm{PC}}=4.6 \mathrm{~Hz}\right), 46.7,53.8,58.1,59.8\left(\mathrm{~d},{ }^{2} J_{\mathrm{PC}}=5.4 \mathrm{~Hz}\right), 75.5\left(\mathrm{~d},{ }^{1} J_{\mathrm{PC}}=\right.$ 166.2 Hz), $164.2\left(\mathrm{~d},{ }^{2} J_{\mathrm{PC}}=20.7 \mathrm{~Hz}\right) ; \mathrm{MS}(\mathrm{EI}): \mathrm{m} / \mathrm{z}(\%) 287$ (4.3), 168 (7.6), 111 (33.3), 98 (100), 84 (19.1), 55 (11.3), 41 (13.6); Anal. Calcd for $\mathrm{C}_{14} \mathrm{H}_{26} \mathrm{NO}_{3} \mathrm{P}: \mathrm{C}, 58.52 ; \mathrm{H}$, 9.12; N, 4.87; P, 10.78. Found: C, 58.43; H, 9.05; N, 4.97; P, 10.84. 


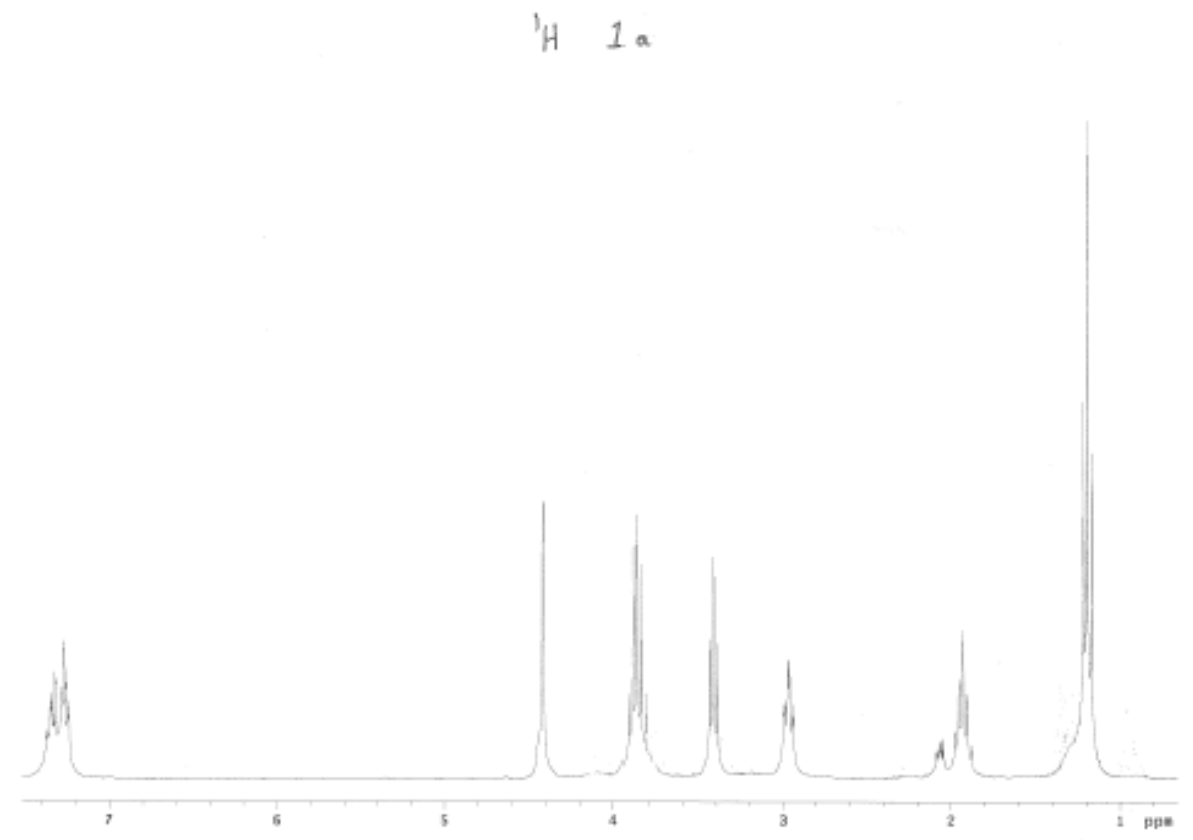

${ }^{13} \mathrm{C} \quad \mathrm{a}$

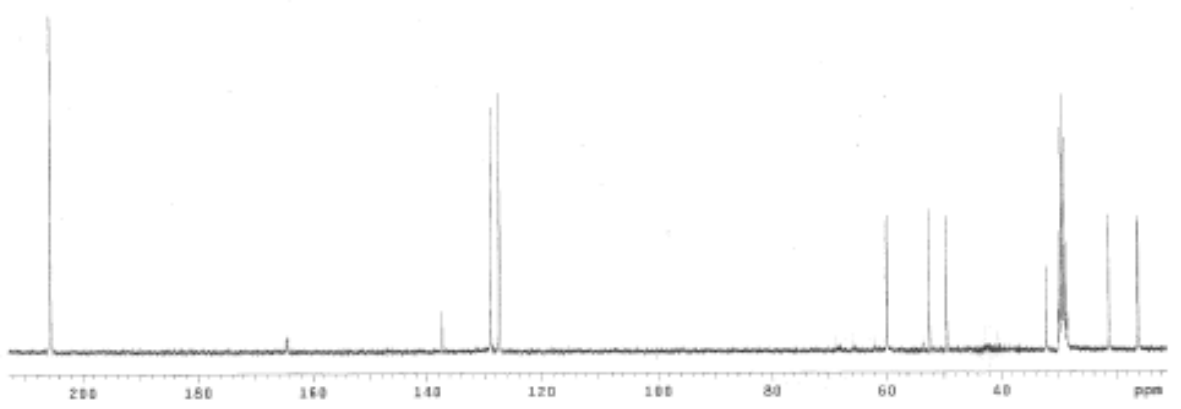


'H 16

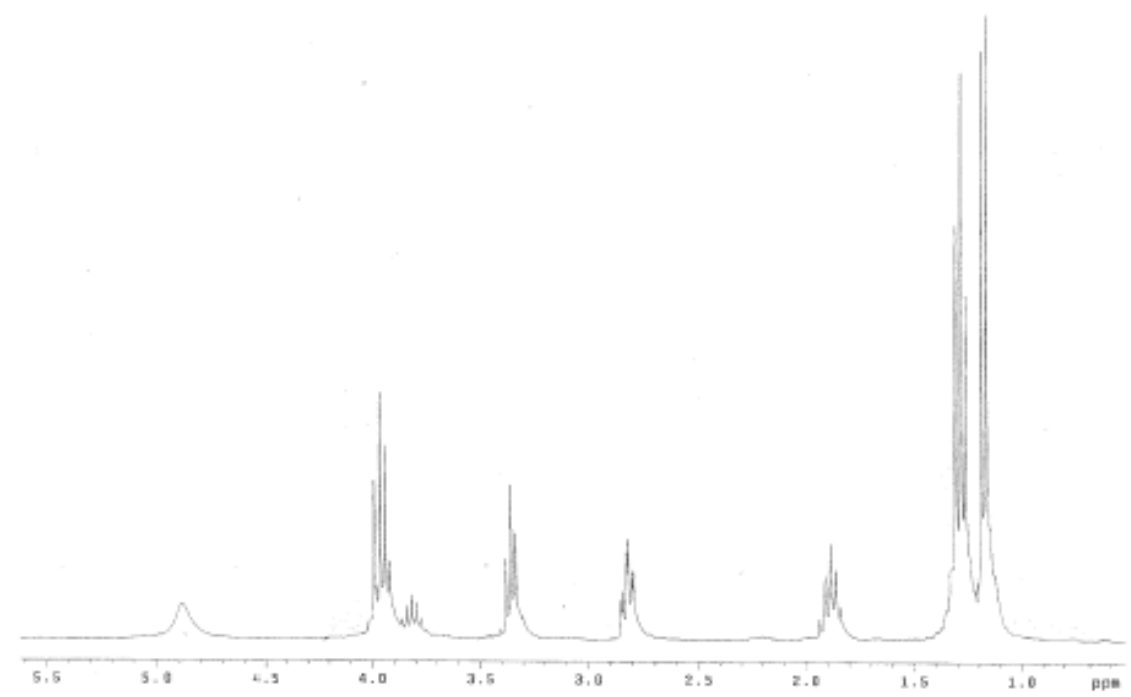

${ }^{13} \mathrm{C}$

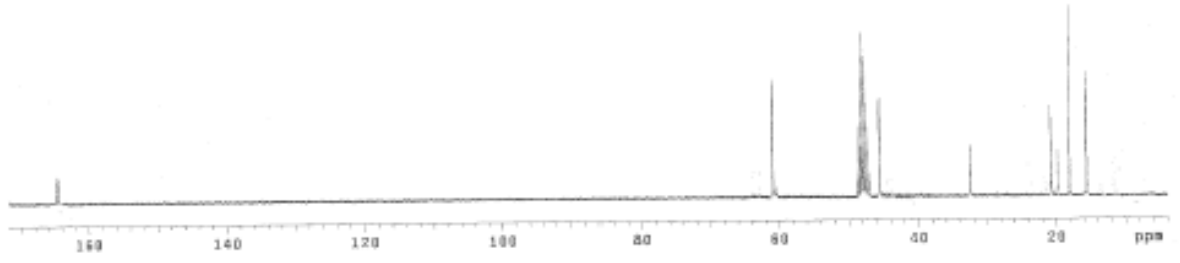


'H $1 \mathrm{C}$

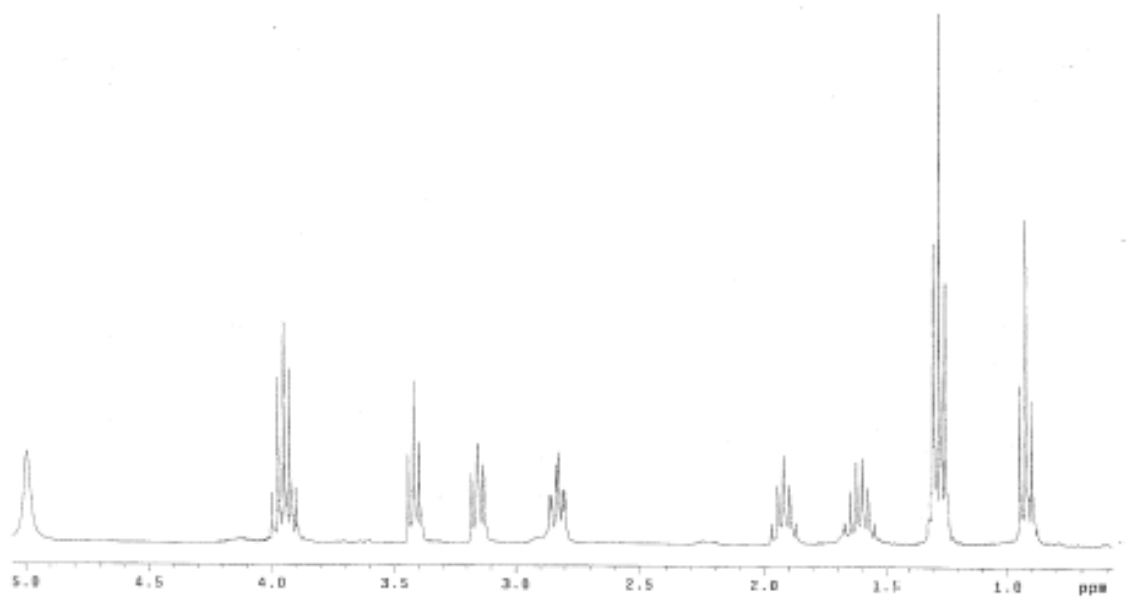

光 $1 \mathrm{C}$

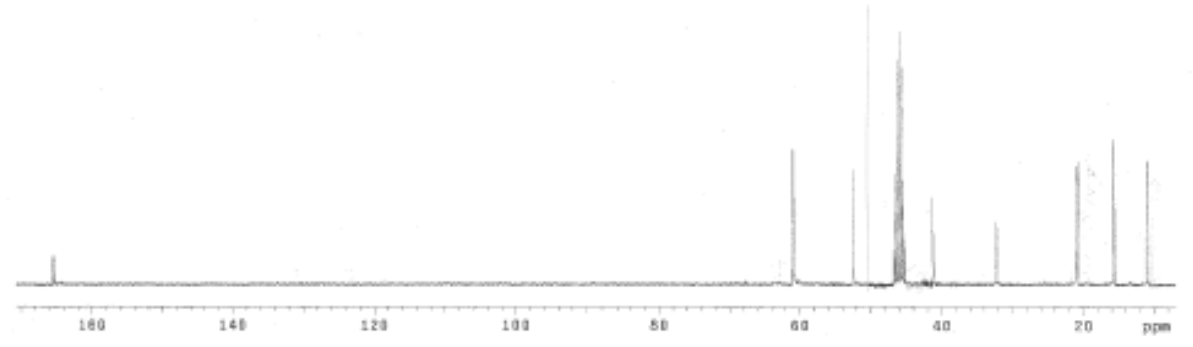


$1 d$

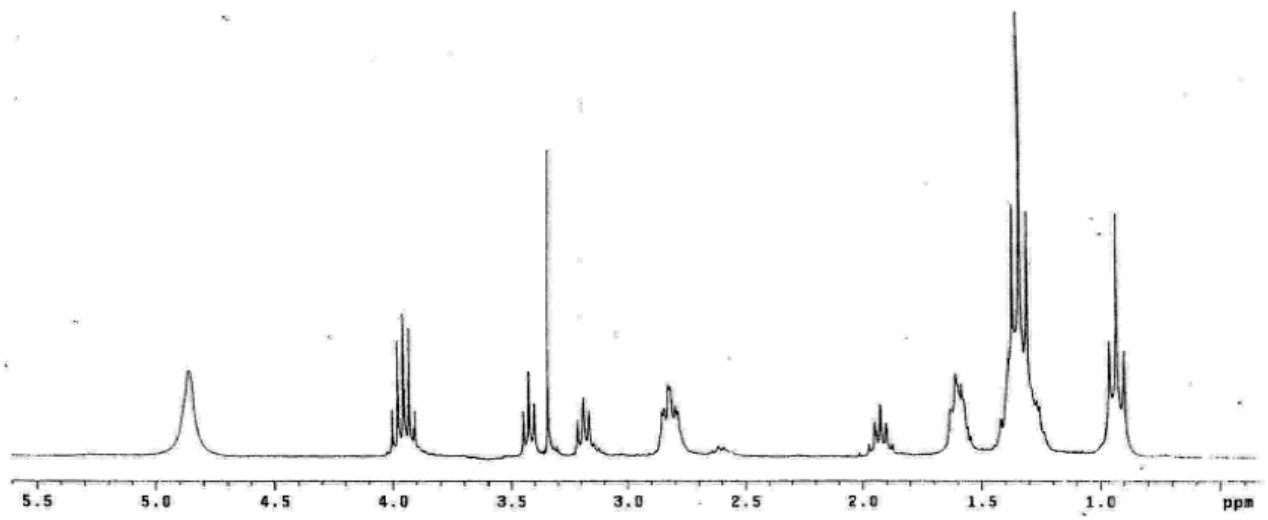

I $1 d$

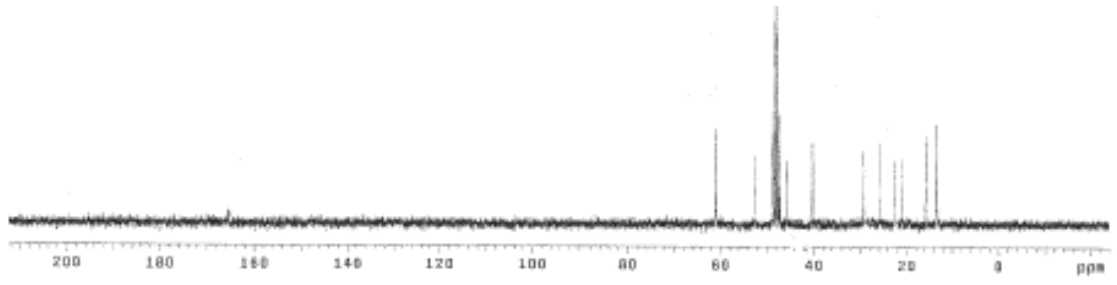


${ }^{1} \mathrm{H} \quad 1 e$

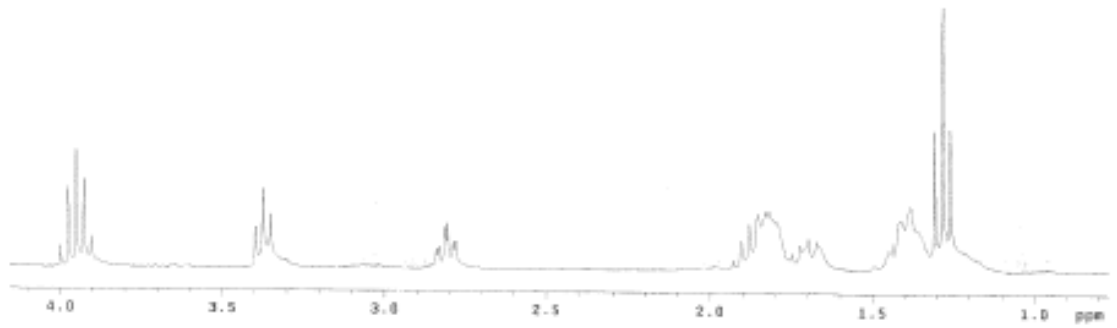

${ }^{13} \mathrm{C} \quad 1 \mathrm{e}$

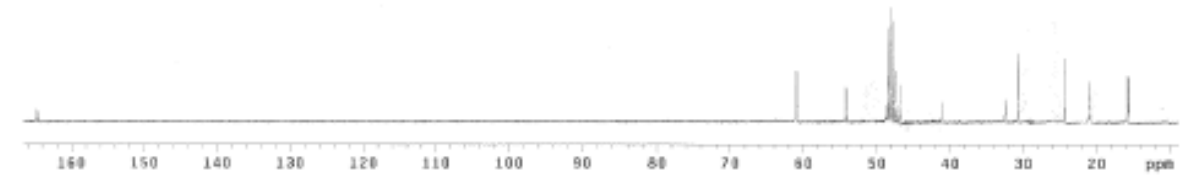


'H $1 f$

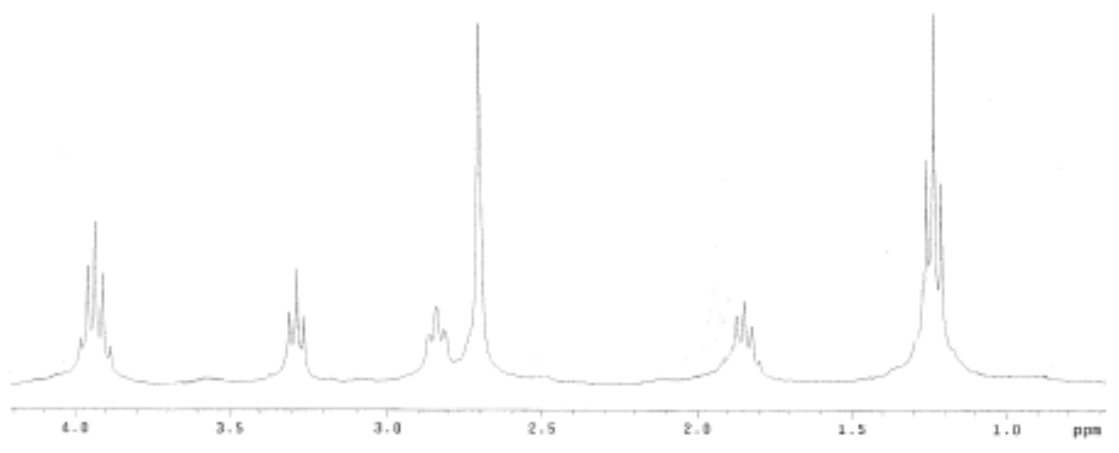

${ }^{13} \mathrm{If}$

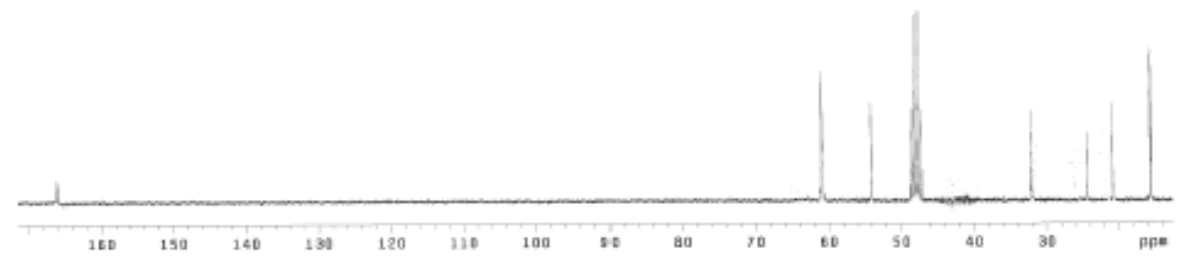


'H 19

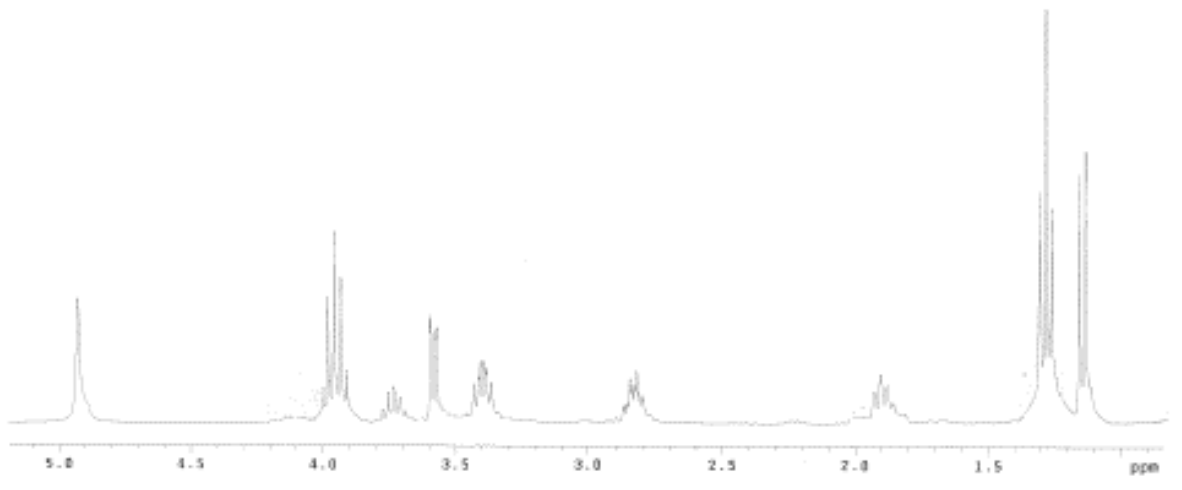

${ }^{13} \mathrm{C}^{1 \mathrm{~g}}$

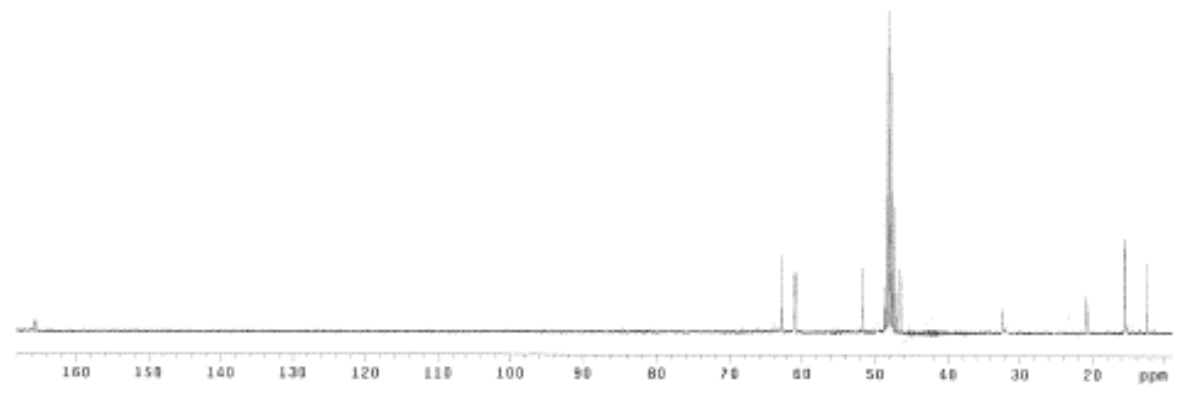


'H $1 \mathrm{~h}$

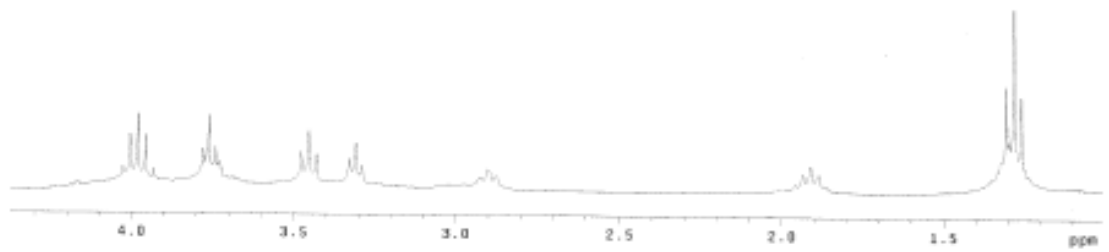

13. $1 \mathrm{~h}$

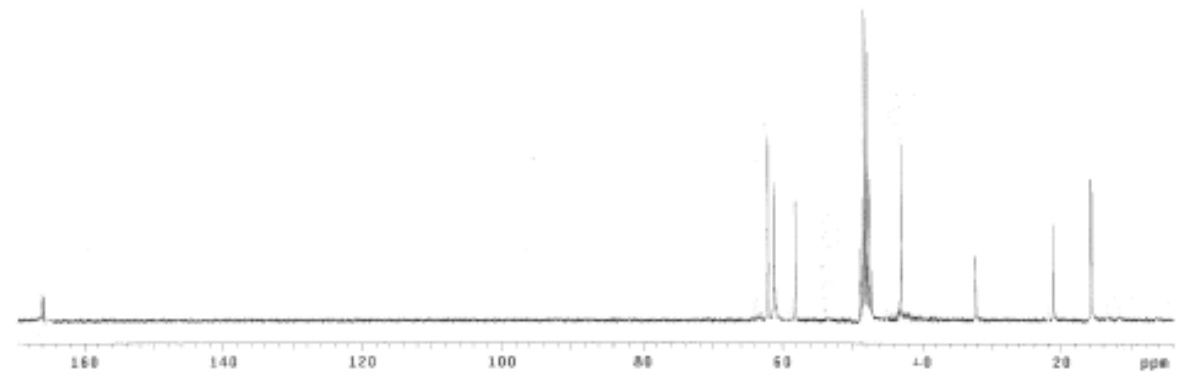




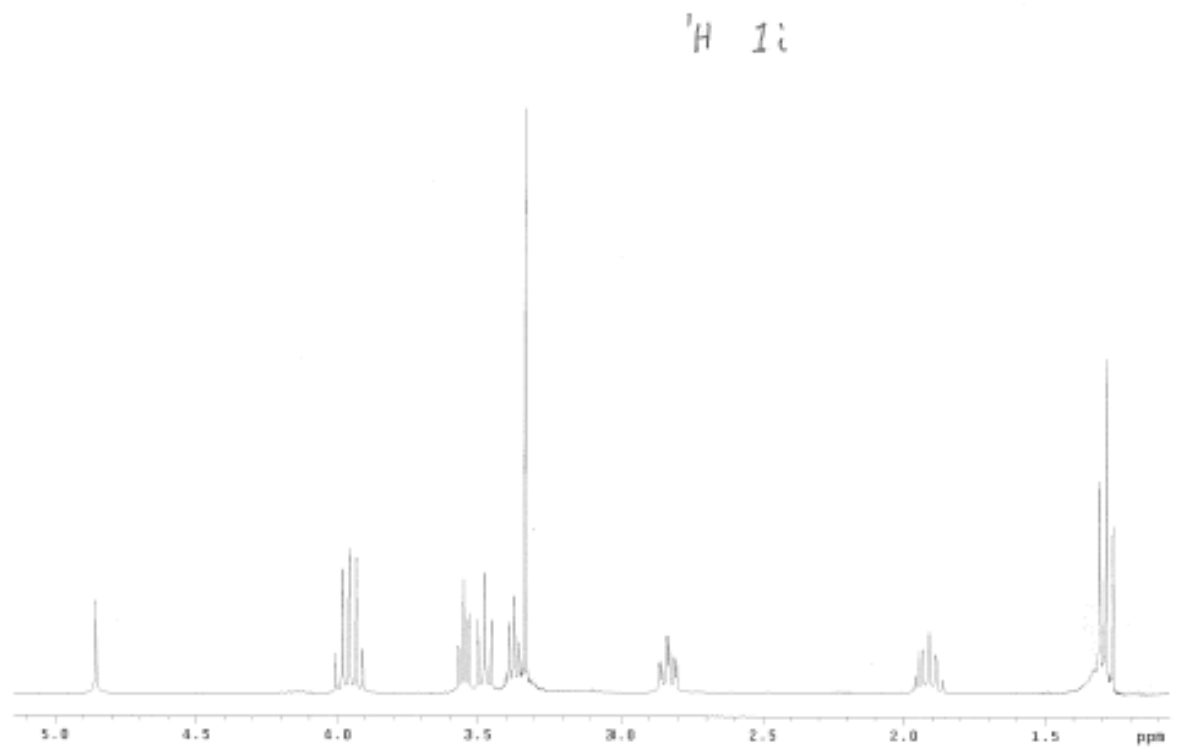

${ }^{13} \mathrm{C} \quad 1 i$

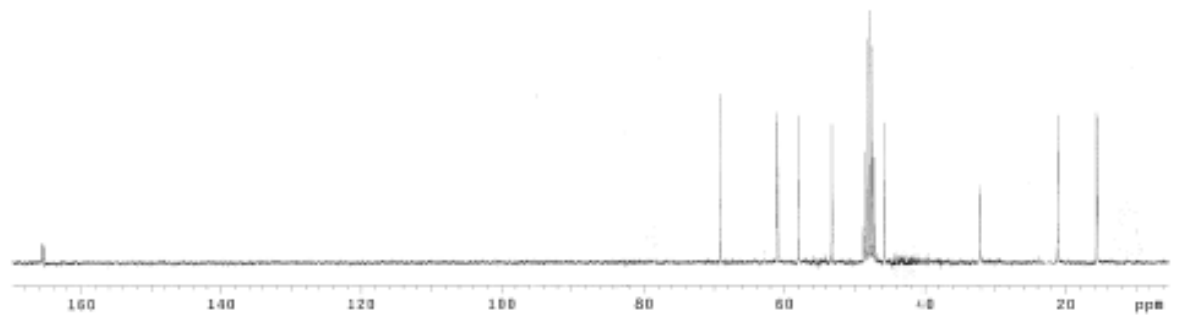


$1 j$
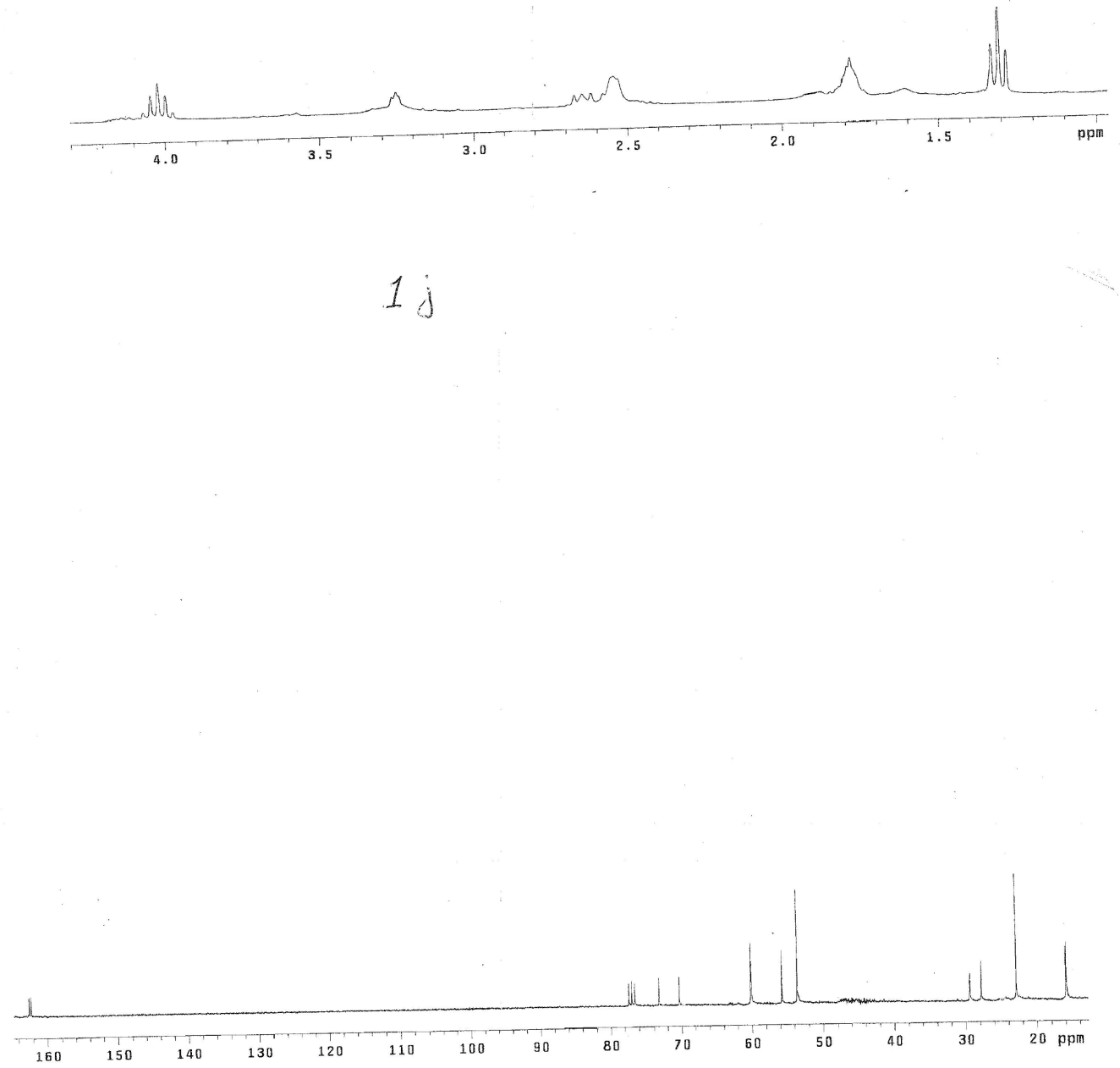
$1 k$

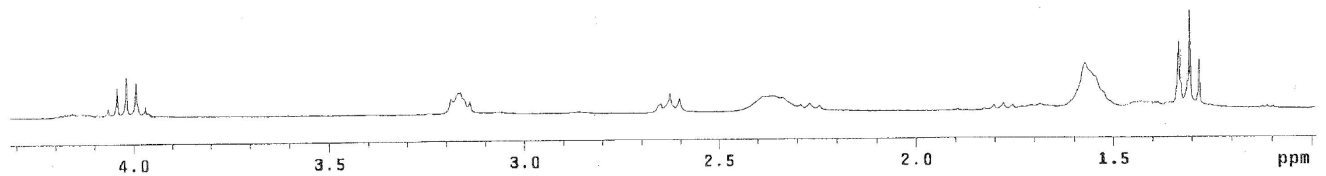

$1 k$

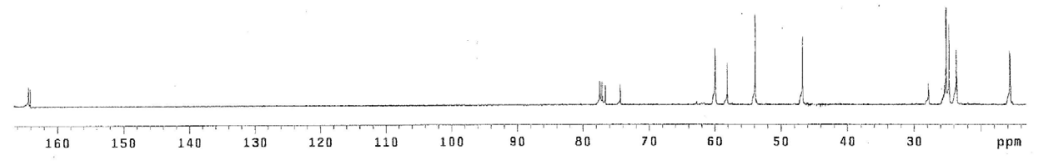

